\title{
Synergetic Light-Harvesting and Near-Field Enhancement in Multiscale Patterned Gold Substrates
}

\author{
Luis A. Guerra Hernández, ${ }^{\dagger}$ María Antonieta Daza Millone, ${ }^{\ddagger}$ Emiliano Cortés, ${ }^{\ddagger}$, Marcos Federico Castez, $^{\ddagger}$ \\ Baptiste Auguié, ${ }^{\dagger, \|}$ María E. Vela, Roberto C. Salvarezza, ${ }^{\ddagger}$ and Alejandro Fainstein ${ }^{*}{ }^{\dagger}$ \\ ${ }^{\dagger}$ Centro Atómico Bariloche e Instituto Balseiro, San Carlos de Bariloche, 8400 Río Negro, Argentina \\ ${ }^{\ddagger}$ Instituto de Investigaciones Fisicoquímicas Teóricas y Aplicadas, INIFTA-CONICET-UNLP, Universidad Nacional de La Plata, \\ 1900 La Plata, Argentina
}

\begin{abstract}
Sphere-segment void (SSV) cavities have emerged as promising substrates for reproducible Surface Enhanced Raman Scattering (SERS), offering strong and uniform electromagnetic enhancement associated with the excitation of cavity-like localized surface plasmon resonances tunable across the UV-vis-near IR range, with a facile largescale fabrication process. High-resolution electron micrographs of these structures reveal a considerable departure from the idealized smooth spherical cavity shape; notably, the electrochemical deposition of gold yields an important surface roughness. We investigate this contribution to the SERS activity of SSV substrates with a series of experiments, varying the degree of surface roughness using thermal annealing and gradual electrochemical roughening. Notably, we observe that both roughness

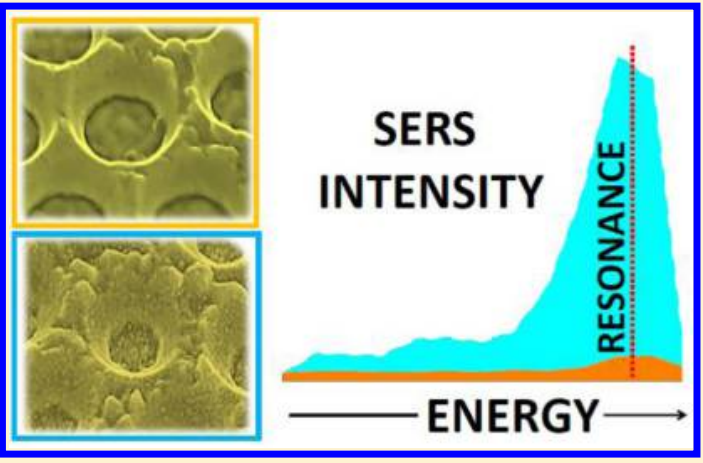
features and cavity-like modes operate in conjunction as a multiscale antenna to provide larger SERS efficiency than the two mechanisms considered separately. We conclude that the main role of the ordered cavity structure is to increase the plasmonic mode density near rough surface features, thus, optimizing the coupling of far-field radiation (light harvesting) to locally enhanced near fields.
\end{abstract}

KEYWORDS: plasmonic substrates, sphere-segment void arrays, roughness, multiscale antennas, surface enhanced raman spectroscopies, sensing

$\mathrm{T}$ he optical properties of gold nanostructures have been the object of intense research activity in the past decades, owing to their promising potential in a variety of technological applications ranging from solar cells, electronics, wave guiding, sensing, display technology, and so on. ${ }^{1,2}$ As light interacts with noble metals, the high density of free conduction electrons may sustain collective oscillations known as surface plasmon resonances, which entail these nanostructures with unique optical properties, such as strongly enhanced scattering and absorption, as well as focusing of incident radiation into localized regions of space with enhanced near-fields. A prime example of plasmonic activity with important practical applications is the field of Surface Enhanced Raman Scattering (SERS), ${ }^{3}$ a powerful and relatively mature material characterization technique that relies heavily on electromagnetic enhancement factors in the vicinity of nanostructured metal substrates. The SERS intensity typically scales with the fourth power of the electric field, and for corrugated surfaces with nanometric gaps and crevices, the enhanced electric field can reach several orders of magnitude in very localized regions. Unfortunately, the largest enhancement factors are generally accompanied by poor spatial homogeneity and lacking reproducibility across substrates. Indeed, one of the major hurdles of surface-enhanced spectroscopies remains the considerable variation in enhancement factors ${ }^{4}$ that precludes quantitative application of the technique toward analytical studies. $^{5}$

In recent years, sphere-segment void (SSV) arrays $^{6-11}$ have emerged as a promising platform to combine uniform field enhancement with good reproducibility and ease of fabrication. They consist of a regular two-dimensional array of spherical voids drawn onto a metallic substrate (Figure 1). The fabrication procedure involves a two-step deposition technique: first, a close-packed hexagonal layer of polystyrene spheres is arranged onto a metal-coated substrate, forming a well-defined mask; second, additional material is deposited through this masking layer via electrodeposition. ${ }^{6}$ The spheres are subsequently removed with appropriate chemicals, leaving the metallic nanostructure exposed. More recently, a variation has been proposed by the Baumberg group, whereby an intermediate mold is produced with polymer/silica opal films, allowing direct transfer of the nanostructure over multiple copies via nanoimprinting. ${ }^{12}$

The motivation of this work is to reach a better understanding of the role of surface plasmon excitations in the Raman scattering enhancement of such substrates, grown with nanosphere templates, and presenting substantial surface

Received: June 22, 2015

Published: August 20, 2015 


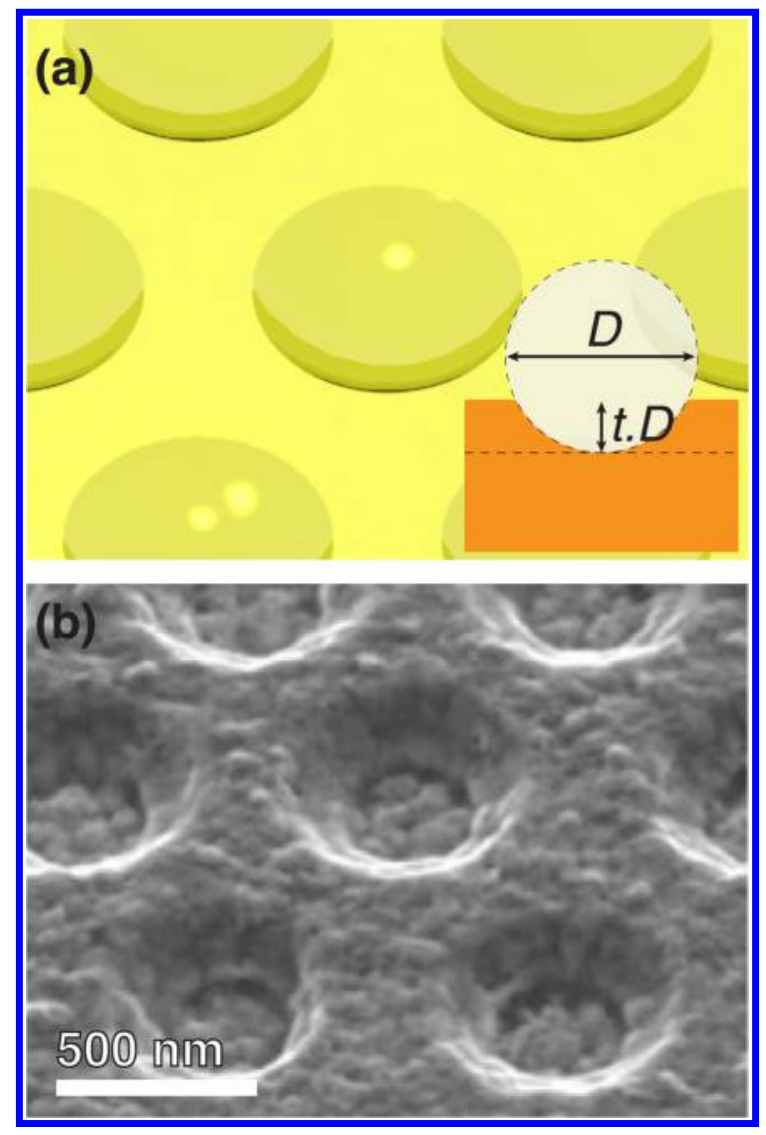

Figure 1. Illustration of imperfections in real SSV substrates. (a) 3D rendering of an ideal SSV structure. The inset schematic defines the geometry and the truncation parameter $t$, with a cross-section through a sphere used as template. (b) High-resolution SEM of a Au SSV array. Sphere diameter $600 \mathrm{~nm}, t=0.15$.

roughness in addition to the regular pattern. To this aim, we will carefully assess previous data on SSV arrays from the existing literature and conduct novel experiments to clearly expose the underestimated role of surface roughness in the SERS response.

SSV arrays have been extensively studied since the original work of Bartlett et al., ${ }^{6-8}$ with detailed studies of the plasmon modes supported by the structure (propagating Bragg plasmons, ${ }^{13}$ and localized Mie-type plasmons $\left.{ }^{11,14-16}\right)$, aided by numerical simulations. ${ }^{14}$ The system was found extremely versatile, with possibilities to tune plasmon resonances from the near UV to the near IR ends of the spectrum by varying the sphere size. The relative contribution of distinct plasmon modes can also be easily tailored for a particular application by varying the truncation parameter $t$ (see Figure 1). SSVs support a rich variety of plasmonic modes, with different field profiles and dispersion. We find, however, that some important features in the data should be analyzed critically, as they depart substantially from the theoretical claims for idealized structures (with perfect symmetry and smooth surfaces). In attempting to correlate spectral reflectivity maps for a variety of samples with observed SERS enhancement factors for a probe molecule uniformly deposited over the cavities, plasmon resonances were seen to have a role in the response, but were not the only factor at play. ${ }^{17,18}$ Indeed, SSV arrays are complex nanostructures presenting numerous unavoidable fabrication defects and intrinsic roughness. Their complex geometry yields many plasmonic excitations that can be hard to capture even with state-of-the-art electromagnetic modeling tools. In this context, detailed and systematic experimental studies are often needed to fully explore the rich optical response of such samples.

The interplay between nanoscale surface roughness and plasmonic modes of larger features is a topic of great practical importance, since practically all fabrication techniques will introduce a residual amount of surface imperfections, yet only few studies have been devoted to this question. Hohenau and co-workers recently concluded, ${ }^{19,20}$ after a series of carefully controlled experiments on simpler geometries, that surface roughness and plasmon resonances may interact in synergy, with a combined near-field enhancement surpassing either effect taken separately. Interestingly, the far-field properties of the substrate may appear relatively unchanged from the ideal (smooth) nanostructure, ${ }^{21,22}$ yet the near-field enhancement experienced by local probes can differ substantially provided conditions are met for the nanoscale roughness to be located in regions of high plasmon mode density. This important conclusion will be used as our working hypothesis throughout the manuscript and motivates the importance of precise structural characterization and surface morphology of SSV arrays with respect to their SERS activity. In fact, studies on films patterned into cavity shapes and made of colloidal gold nanoparticles suggested the existence of an increased electromagnetic enhancement, presumably caused by the combination of optical interactions on two different scales: surface plasmonenhanced near-fields due to nanoscale features and far-field photonic coupling by periodic structures at the microscale. ${ }^{23}$ Recently, the incorporation of $\mathrm{Au}$ nanoparticles into the voids of SSV arrays has been explored, showing also an important increase in field enhancement at the particle-cavity junction. ${ }^{24-26}$ These results reinforce the idea that electromagnetic fields in SSV arrays may be further confined by smaller metallic features at the nanometric scale.

If a small but nonvanishing amount of roughness participates in the SERS response of these cavity substrates, we anticipate that there may be an optimum level of roughness, which may be varied with the deposition process. This important question will thus be first addressed with experiments on unpatterned gold substrates, treated with progressive amounts of electrochemical roughening. Once the effect of purposely introduced roughness is independently established, we will proceed to study its effect on Au SSV arrays. We will show, by detailed studies as a function of truncation of the SSV array, the existence of strong SERS resonances tuned with cavity-like plasmons of the structure, thus, clearly demonstrating efficient light-harvesting by these microscale metallic antennas. In addition, by purposely tailoring their roughness, either increasing it by electrochemical methods or decreasing it by controlled annealing, we demonstrate the multiplicative strong contribution to the field enhancement by structures at the nanoscale. Detailed surface studies by STM and image processing techniques quantify the amount and characteristics of the introduced roughness, identifying the structures potentially involved in the observed combined multiscale performance of these SERS substrates.

\section{RESULTS AND DISCUSSION}

Role of Roughness in SERS with Unpatterned Gold. In order to correlate the effect of surface roughness in SSV arrays, we started with experiments on a flat, unpatterned gold surface. Increments in area up to 100 times can be readily obtained via electroreduction of Au oxide formed during variable times of an 
anodization procedure (Figure 2a). This study has been complemented by a detailed surface characterization with atomic force microscopy (AFM) and SERS.

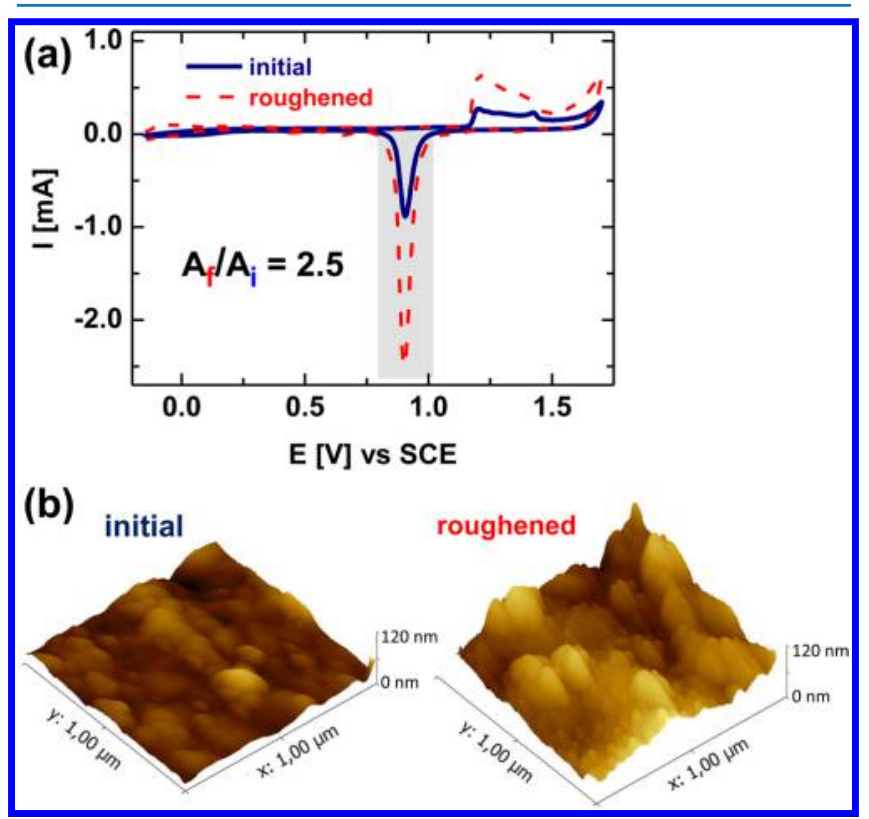

Figure 2. (a) Cyclic voltammograms of a Au wire in $0.5 \mathrm{M} \mathrm{H}_{2} \mathrm{SO}_{4}$ at $\nu$ $=0.1 \mathrm{~V} \cdot \mathrm{s}^{-1}$ before (solid blue curve) and after (dashed red curve) electrochemical roughening, yielding a $2.5 \times$ increment in surface area. (b) AFM images of a typical area for the initial Au surface (left) and after electrochemical roughening (right).

Scanning electron microscopy (SEM) images of the overlayer cross-section $^{27}$ and AFM images of the surface (see Figure 2b) indicate that the high-area nanostructured gold presents a columnar-like structure containing a large density of pores. Ellipsometric data have shown that the density of the electrochemically prepared nanostructured gold is half that of pure gold. $^{28,29}$ Enhanced stability of thiolate self-assembled monolayers (SAMs) on these nanostructured substrates also suggests the presence of a large number of defects. ${ }^{30}$

Effect of Roughness on SERS and Evidence for Surface Relaxation. In Figure 3 we summarize our SERS measurements for substrates with a varying amount of surface roughness. The amount of roughness is quantified by the relative change of surface area, $R=A_{\mathrm{f}} / A_{\mathrm{i}}$, with $A_{\mathrm{i}}$ the initial area, and $A_{\mathrm{f}}$ its value after the electrochemical roughening procedure. The typical spectrum of 4-mercaptobenzoic acid (MBA) is shown for reference in the top panel. The strong Raman band at 1076 $\mathrm{cm}^{-1}$, associated with $v 12$ aromatic ring vibrations, ${ }^{31}$ was monitored for different samples, and the integrated intensity is presented in the bottom panel as a function of increased surface area (each point is the average of at least five measurements at different spots, the error bars indicate their maximum dispersion). Smooth wires (without roughening procedure) do not exhibit any Raman peak in the spectra recorded. We considered two situations: first, the electrochemically roughened surface was allowed to relax in the acid solution, rinsed with ethanol, and immersed in a $1 \mathrm{mM}$ MBA ethanolic solution; second, the freshly electro-roughened surface was rinsed and its structure quickly frozen using MBA molecules, thus, limiting considerably the surface relaxation prior to SERS characterization. These two initial conditions yield a vastly different SERS response at high rugosity, as shown in Figure $3 \mathrm{~b}$.

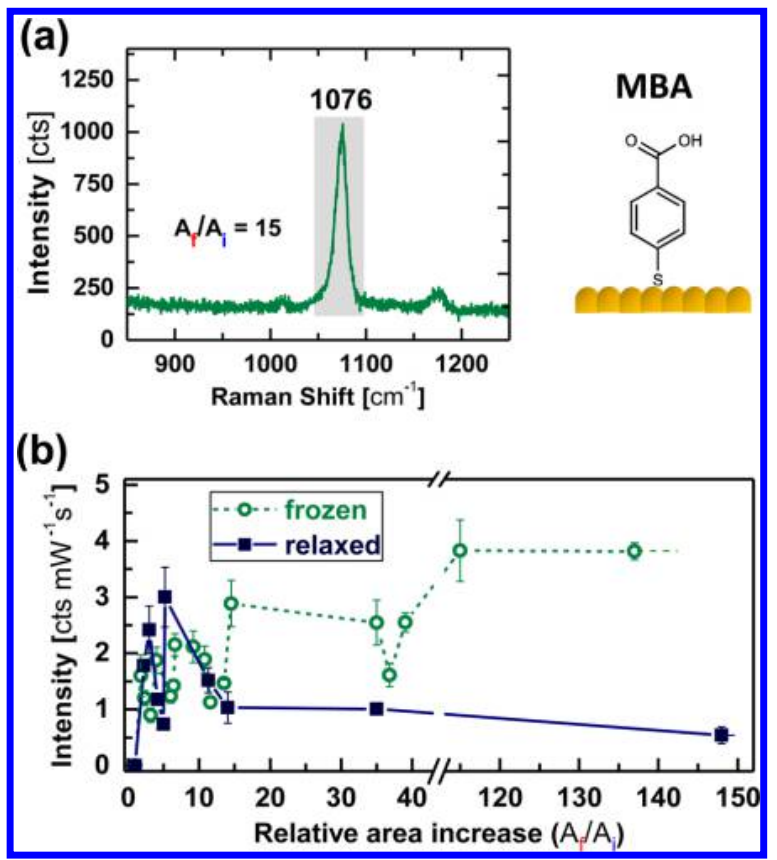

Figure 3. (a) SERS spectrum of mercaptobenzoic acid (MBA) adsorbed on a rough Au surface with $R=15$. (b) Evolution of the intensity of the $1076 \mathrm{~cm}^{-1}$ Raman band, normalized by laser power and acquisition time, as a function of surface roughness. The solid symbols (solid line) correspond to experiments performed on a "relaxed" surface, while the open symbols (dashed line) are for a "frozen" sample, limiting surface relaxation. The error bars correspond to the maximum dispersion of values for at least five different positions across the sample.

From these data, confirmed with a number of repeated experiments, we note the following trends. First, a considerable sample-to-sample variation is observed, as visible in the relatively scattered data for low surface area. This suggests that the level of control in electrochemical roughening of such substrates is not sufficient to guarantee reproducible and homogeneous enhancement factors in SERS, and variations of a factor of 2 are to be expected with this type of fabrication method. Second, even the lowest level of surface roughness appears to produce substantial increase in SERS intensities, with a peak at $R \sim 5-10$. Beyond this initial regime of low rugosity, we need to differentiate the two cases corresponding to the previously relaxed surface (solid symbols) or the thiolfrozen surface (open symbols). At high rugosity, the surface presents nanostructures of high aspect ratio, in the form of deep crevices and heterogeneous nanopilars. These features are associated with a large increase in surface free energy and are, thus, thermodynamically unstable. Relaxation of the surface toward smoother surfaces with lower curvature gradients occurs naturally at room temperature in short time scales, as we shall discuss below. The chemisorption of thiol molecules immediately after electrochemical treatment partially stops this surface restructuration. ${ }^{30} \mathrm{We}$ observe a relatively stable SERS intensity at higher surface area, suggesting that additional rugosity is not useful for surface-enhanced spectroscopy. Detailed structural characterization with high-resolution scanning electron microscopy reveals deeper structures that penetrate hundreds of nanometers inside the bulk sample, ${ }^{27}$ thus, poorly accessible to incident light. This apparent saturation is not seen for unthiolated surfaces, where surface relaxation occurs in the time frame of minutes to hours and 
cancels the effect of high initial rugosity. We will discuss next a model for the evolution of a high rugosity 2D interface.

Mullins Model of Interface Evolution. The surface diffusion is often an important mass-transfer process that affects the evolution of the surface morphology at the nanoscale. It is particularly important for metallic nanostructures, that suffer drastic morphological changes at relatively low temperatures ${ }^{32}$ and even at room temperature. ${ }^{33}$

The continuous theory of interface evolution mediated by surface diffusion is a well-established topic after the pioneering work of Mullins. ${ }^{34,35}$ In this framework, a given interface enclosing an isotropic sample evolves according to the Mullins equation

$$
v_{\mathrm{n}}=-K \Delta_{\mathrm{S}} C
$$

where $v_{\mathrm{n}}$ is the normal velocity at a given point on the evolving surface, $\Delta_{S}$ is the intrinsic surface Laplacian (the so-called Laplace-Beltrami operator), and $C$ is the local curvature. The coefficient $K$ depends both on the type of material considered and on the temperature through the relationship $K=\frac{\gamma D_{s} \Omega^{2} \nu}{k_{\mathrm{B}} T}$, where $\gamma$ is the surface tension, $D_{s}$ is the diffusion constant, $\Omega$ is the atomic volume, $\nu$ is the adatom density on the surface, $k_{\mathrm{B}}$ is the Boltzmann constant, and $T$ is the absolute temperature.

For high-surface roughness, depending on the specific initial condition, the surface diffusion flow dictated by eq 1 can promote diverse morphological changes at interfaces, including the formations of necks, coalescence of structures, formation of voids, tunnels, and overhangs, and so on. In fact, the Mullins equation is a fourth order nonlinear equation that can produce a rather intricate surface evolution. To give a simple example of such phenomena, let us consider Figure 4, where we draw the evolution of a 2D interface obtained from a numerical integration of eq 1 for an initial condition (Figure 4a) consisting of a sample with a certain distribution of rectangular wells with different sizes and aspect ratios. The coefficient $K$ used in the simulation was $K=10^{3} \mathrm{~nm}^{4} / \mathrm{s}$, a value typical for metals at temperatures around $100{ }^{\circ} \mathrm{C} .^{36}$ Overhangs can be appreciated in (b), void formation and coalescence of structures are clearly seen in (c), (d), and (e), while the coexistence of equilibrium structures (circular voids) with nonequilibrium voids is evident from (f). Considering the qualitative properties of this simple $2 \mathrm{D}$ simulation, we can readily infer that the surface diffusion of a 3D spongy structure, will likely yield a very complex morphological evolution with a nonuniform distribution of voids, tunnels, and metastable transient structures. Evidently, some of such structures will be partially, when not totally, occluded to the incident light.

On the other hand, for an initial state of lower surface roughness other parameters must be considered, like the Ehrlich-Schwoebel barrier. ${ }^{37,38}$ Diffusion barriers at grain boundaries prevent a complete surface relaxation of the surface. ${ }^{39}$ Consequently, a complete suppression of the surface roughness at the nanoscale might never be achieved at room temperature and under practical time scales. We thus conclude that, for a typical experiment lasting a few hours, an irreducible component of nanometer-scale roughness does not relax and contributes to SERS and that higher levels of initial rugosity will not improve the SERS signals, unless special stabilizing agents (here, thiol molecules) are used to "freeze" the surface.

Following the results of this section on unpatterned gold substrates, we now turn to SSV arrays, where in addition to the intrinsic surface roughness associated with the electrodeposi-

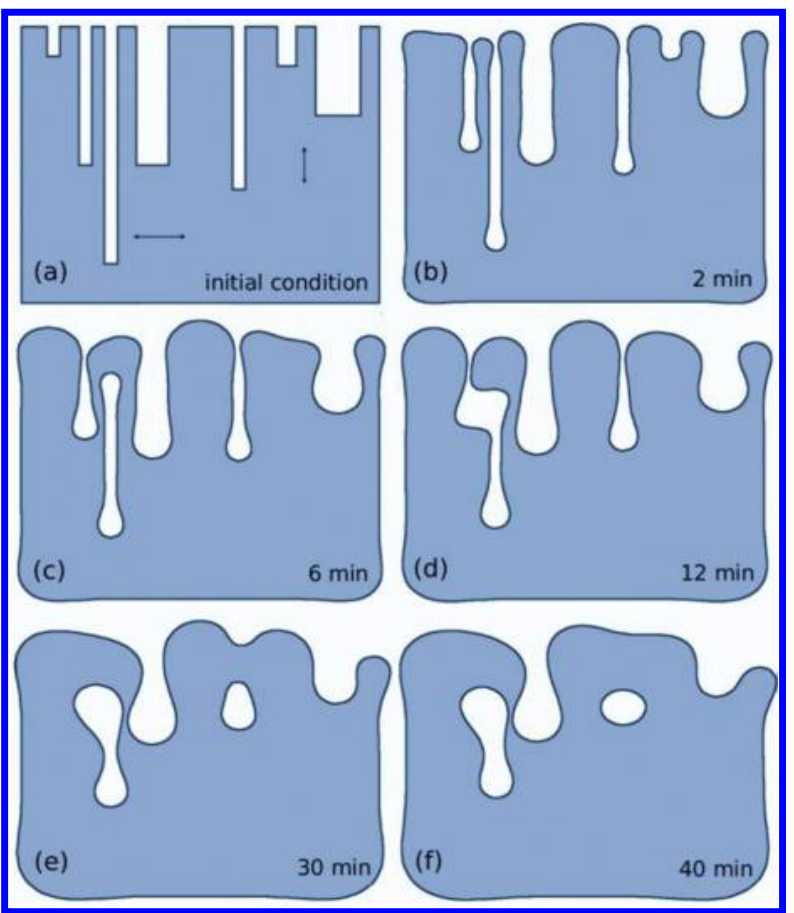

Figure 4. Simulated snapshots of surface relaxation obtained by numerical integration of the Mullins equation (eq 1), with $K=10^{3}$ $\mathrm{nm}^{4} / \mathrm{s}$. Starting from an initial 2D structure composed of sharp corners of varying size and geometry, the evolution of the protuberances and holes due to surface diffusion progressively yields a smoother surface, with the possible development of internal voids isolated from the surface. The arrows in the top left panel correspond to $100 \mathrm{~nm}$.

tion process, a variety of plasmon modes strongly influence the optical response in both near-field and far-field experiments.

Effect of Surface Roughness in SSV Arrays. As reviewed in the introduction, SSV arrays sustain a variety of plasmon modes, with frequencies that can be tuned across the visible-IR range varying the sphere diameter or the truncation height. In the current work, we are interested in the possible interaction, as evidenced in SERS intensities, between such plasmon modes and intrinsic surface roughness. As discussed in the previous section, a residual component of roughness does not fully relax and should therefore be present in SSV arrays. Likewise, we will vary the initial rugosity by electrochemical roughening. An illustration is shown in Figure 5 with high-resolution SEM images of a cavity before (as-grown) and after electrochemical roughening.

The SERS characterization of as-grown substrates covered with MBA was complemented by subsequent cleaning of the substrate and electrochemical roughening. SEM images of typical samples are presented in Figure 5, together with cyclic voltammograms showing the increase in surface area. For reference, a region with the bare Au substrate was left on the side of the self-assembled polystyrene spheres. The electrodeposition with the same truncation sequence as the SSV array structure was performed also in this unpatterned region, which was also later electrochemically roughened together with the ordered cavity array. In this way the SERS response of the SSV arrays could be compared to that of planar substrates with roughness originated either from the electrodeposition process or purposely increased by electrochemical procedures.

SERS on Roughened SSV Arrays. We summarize in Figure 6 the results of these experiments on SSV arrays. Figure 6a shows 


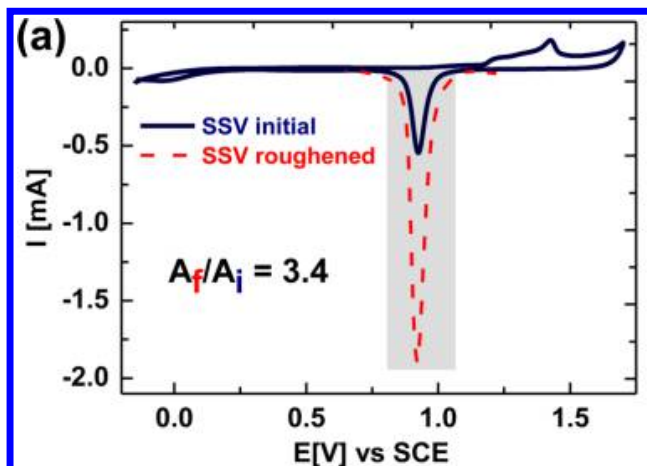

(b) Ssv initial

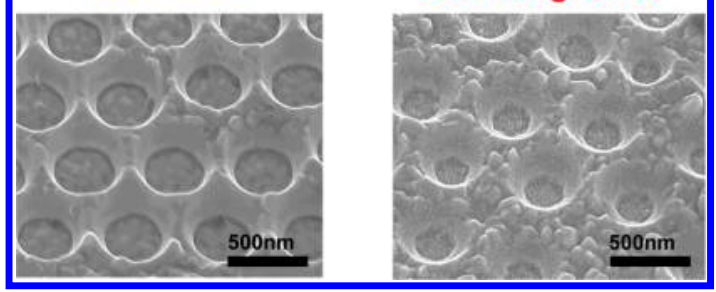

Figure 5. Surface morphology of Au SSV arrays $(600 \mathrm{~nm}$ in diameter, $t$ $=0.27$ ). (a) Cyclic voltammogram at $0.1 \mathrm{~V} / \mathrm{s}$ in $0.5 \mathrm{M} \mathrm{H}_{2} \mathrm{SO}_{4}$ before (solid blue line) and after (dashed red line) electrochemical roughening. (b) Corresponding SEM images, showing the surface topography before and after the roughening procedure $(R=3.4)$.

the reflectivity measured with TE and TM polarizations for the same SSV substrate, as-prepared and later roughened $(R=6)$, for varying truncation steps. For the roughened case, the curves for higher truncation have been multiplied by two for clarity (strong diffuse reflection of light is observed with the naked eye in this situation, corresponding to a reduction in specular reflection). Quite similar spectra are observed with TE and TM polarization. Although essentially the same modes can be identified in the two cases, TM measurements provide clearer traces and will therefore be used for our discussion. While significant changes occur upon roughening, it is clear that the plasmonic response is still present. This is clearly due to the fact that the basic SSV structure is essentially conserved (see Figure $5)$. Note the truncation $(t)$ values at which the laser wavelength crosses the ${ }^{1} \mathrm{P}$ Mie-type mode (highlighted by solid and hollow points). At around the laser wavelength, the main effect seems to be a slight red-shift of the plasmon modes upon roughening, indicated in the left panel of Figure 6 with guides to the eye. At longer wavelengths and for the roughened sample, the identification of the modes becomes less clear. We have also observed that the details of this latter region are also more sample dependent, with different features observable in repetitions of the same experiment.

Figure $6 \mathrm{~b}$ summarizes the corresponding SERS experiments. Data as a function of truncation are shown for the as-grown and roughened SSV arrays, together with the flat Au substrate region that followed the same electrodeposition and roughening procedures. In the latter case, only results for the roughened sample are shown, the flat initially electroplated region did not lead to observable Raman spectra under the same experimental conditions (laser power and acquisition time). Several important features can be highlighted: (i) A strong SERS enhancement is observed for truncation steps close to the resonant crossing between the incident laser wavelength and the dispersion of the ${ }^{1} \mathrm{P}$ Mie-type plasmon

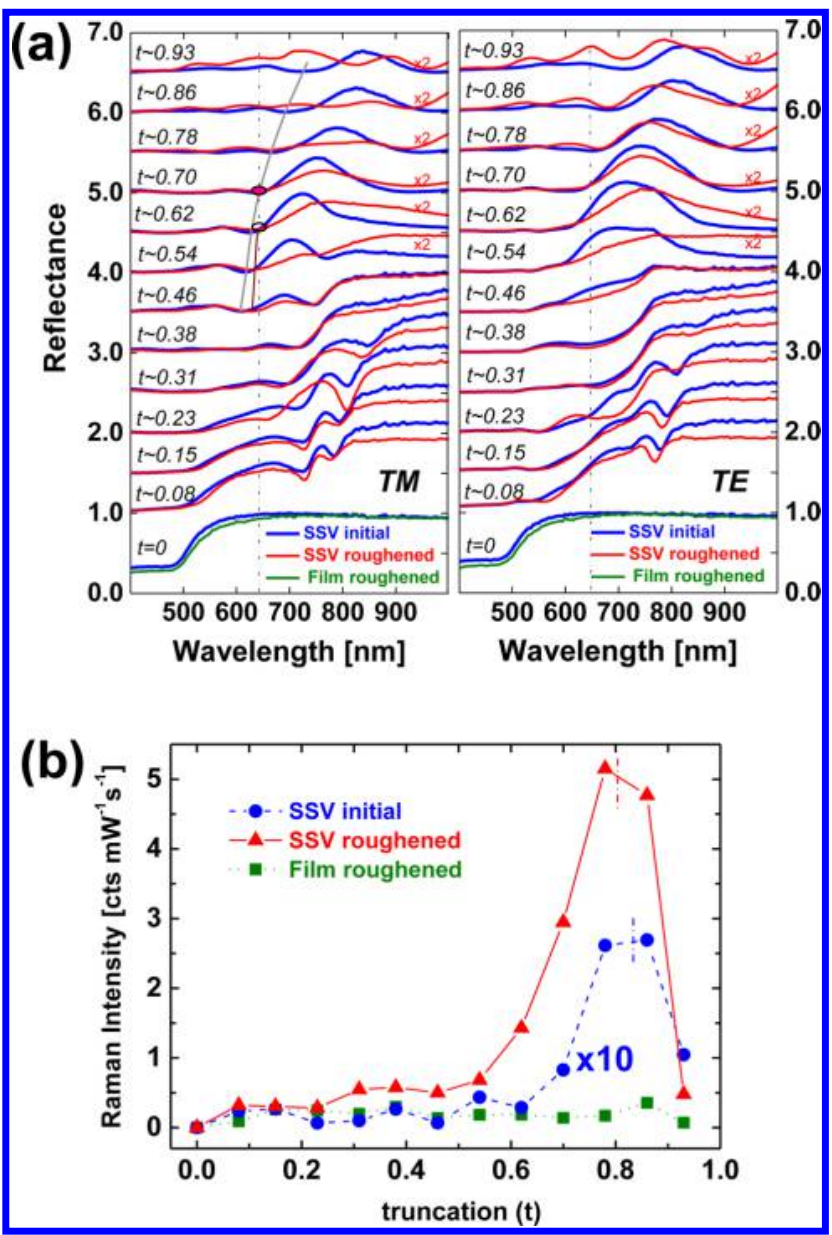

Figure 6. (a) Reflectivity characterization of as-prepared Au SSV arrays (blue, $600 \mathrm{~nm}$ sphere template) and after electrochemical roughening (red, $R=6$ ). The truncation parameter was varied between $t=0$ (unpatterned Au surface) and $t=0.93$ (almost full coverage of the sphere template). Reflectivity curves are vertically offset by steps of 0.5 for clarity. The dispersion of the ${ }^{1} \mathrm{P}$ Mie-type mode is superimposed as a guide to the eye. The values at which the laser wavelength crosses the mode dispersion are highlighted by solid and hollow points. (b) Raman intensity of the $1076 \mathrm{~cm}^{-1}$ band as a function of truncation $t$, with $647 \mathrm{~nm}$ incident light and TE polarization. Integrated intensities for the as-prepared sample (blue circles) were scaled up by a factor of 10 to facilitate comparison with the corresponding intensities for the rough sample (red triangles). Green squares correspond to data collected at the side of the SSV array, in flat regions without polystyrene-assembled spheres, that followed the same electrodeposition and posterior electrochemical roughening procedure.

modes (indicated with open and solid circles in Figure 6a). This unambiguously demonstrates the involvement of the micron-scale cavity-like features of the SSV array as efficient light-harvesting far-field antennas. (ii) The resonant character of this SERS enhancement is conserved for the roughened SSV arrays, displaying increased amplifications (a factor of 20) as compared to the as-grown cavity substrates. The combined effect of far-field light focusing by the SSV array and near-field enhancement by the introduced surface nanostructuring is, thus, clearly evidenced. (iii) A slight shift of the resonance toward smaller truncation levels is observed in the latter case, something that is compatible with the small red shift of the plasmon modes observed upon roughening in Figure 6. (iv) While SERS signals are observed for the nonpatterned $\mathrm{Au}$ 


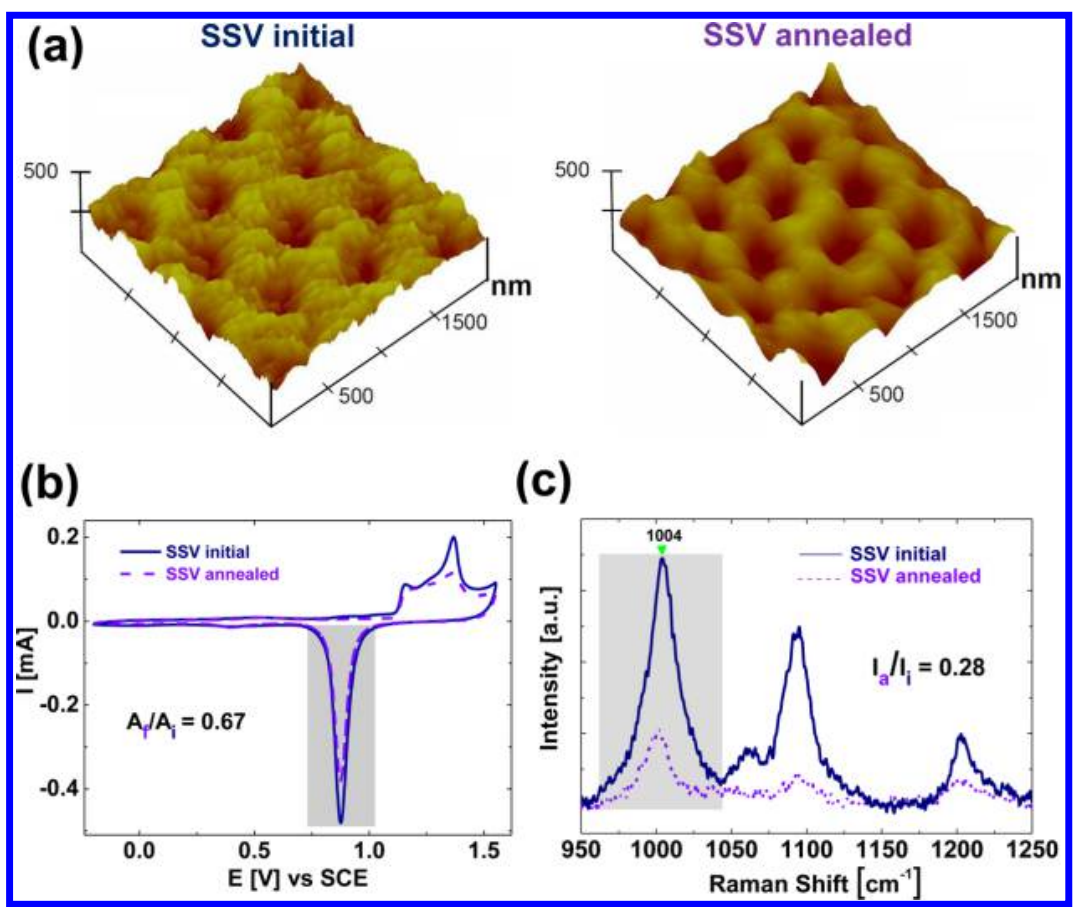

Figure 7. (a) STM images of a Au SSV array as prepared (left) and after thermal relaxation (right) at $500{ }^{\circ} \mathrm{C}$ for 90 min. (b) Voltammograms for the SSV substrate in $0.5 \mathrm{M} \mathrm{H}_{2} \mathrm{SO}_{4}$ electrolyte solution before (solid curve) and after (dashed curve) the thermal annealing process. A reduction of $33 \%$ in surface area can be appreciated by integrating the charge involved in the reduction of the Au oxide. (c) SERS measurements of a 4mercaptopyridine (4MPy) self-assembled monolayer on the SSV array before (solid curve) and after (dashed curve) the thermal annealing process. By integrating the $1004 \mathrm{~cm}^{-1}$ band, a reduction of more than $70 \%$ in the SERS signal is observed.

regions that were first electrodeposited and then electrochemically roughened, this enhancement is essentially independent of the height of initial deposition, and much weaker than for the SSV arrays. (v) The 20-fold increase in the Raman signal after roughening the SSV array cannot be accounted for by the 6-fold increase in the surface area $(R=6)$ but must originate from additional near-field enhancement contributing to the overall SERS response. The 6-fold increase in area can be understood as an upper limit for the expected increase of SERS signal originated in a larger number of immobilized molecules, in the view that not necessarily all the new binding sites are accessible to the laser light.

Thermal Annealing of SSV Arrays. As further confirmation of the role of surface roughness in the SERS response of SSV arrays, we also set out to decrease the residual roughness intrinsic to the electrodeposition, via a thermal annealing process. After exposure of SSV arrays at $500{ }^{\circ} \mathrm{C}$ during $90 \mathrm{~min}$, Scanning Tunneling Microscopy (STM) images showed an evident smoothing of surface features without global changes in the SSV structure (Figure 7a).

After such soft thermal annealing we measured a reduction of $33 \%$ in the total exposed area, Figure $7 \mathrm{~b}$. Comparatively, in the exposed area the SERS intensity of 4-mercaptopyridine (4MPy) decreased by more than $70 \%$, Figure $7 \mathrm{c}$. This decrease in intensity cannot be associated only to a decrease in the exposed area and is, therefore, strongly indicative that the electromagnetic enhancement factor of the SSV substrate was decreased by the annealing process, thus, corroborating the important contribution of nanoscale roughness to the enhancement mechanism in SSV arrays.

Multiscale Contributions to SERS in Gold SSV Arrays. As is readily observable in the images shown in previous sections (Figures 1, 5, and 7), in SSV arrays coexist structures of two different scales, namely, (i) the periodic cavity array (dimensions of the light wavelength) and (ii) much smaller and disordered pointed cusps with dimensions in the range of tens of nanometers. Moreover, it is evident from Figures 5 and 7 that these latter smaller features are particularly sensitive to electrochemical roughening and annealing, with clear consequences on the SERS efficiency. It is therefore of interest to better characterize these features and their relation to the observed SERS performance. It is important to note at this point that experiments trying to decrease (increase) even further the nanoscale features by annealing (respectively, electrochemistry) techniques lead to a partial destruction of the cavity arrays.

The SERS activity of a rough metal substrate is intimately linked to its precise morphology at the nanoscale; this relation has been the subject of intense research since 1974, following the first observation of strong Raman enhancement by Fleischmann and co-workers. ${ }^{40-42}$ Localized plasmon resonances were soon recognized as playing a major role in the electromagnetic enhancement, and the connection between geometry and near-field optics became one of the cornerstone of research in plasmonics. ${ }^{43,44}$ In the context of our experiments, the complex morphology of crevices and protuberances obtained by electrodeposition and subsequent electrochemical roughening makes a direct comparison with theoretical predictions very challenging, and we will thus limit ourselves to a qualitative description based on existing literature. Such rough plasmonic nanostructures present a complex character, whereby a wide variety of pits and cusps coexist in a given area, with close separations. As noted by several authors, ${ }^{4-47}$ the clear experimental evidence that surface roughness helps SERS activity is not trivially linked to a characteristic surface roughness, at least in rough substrates of 


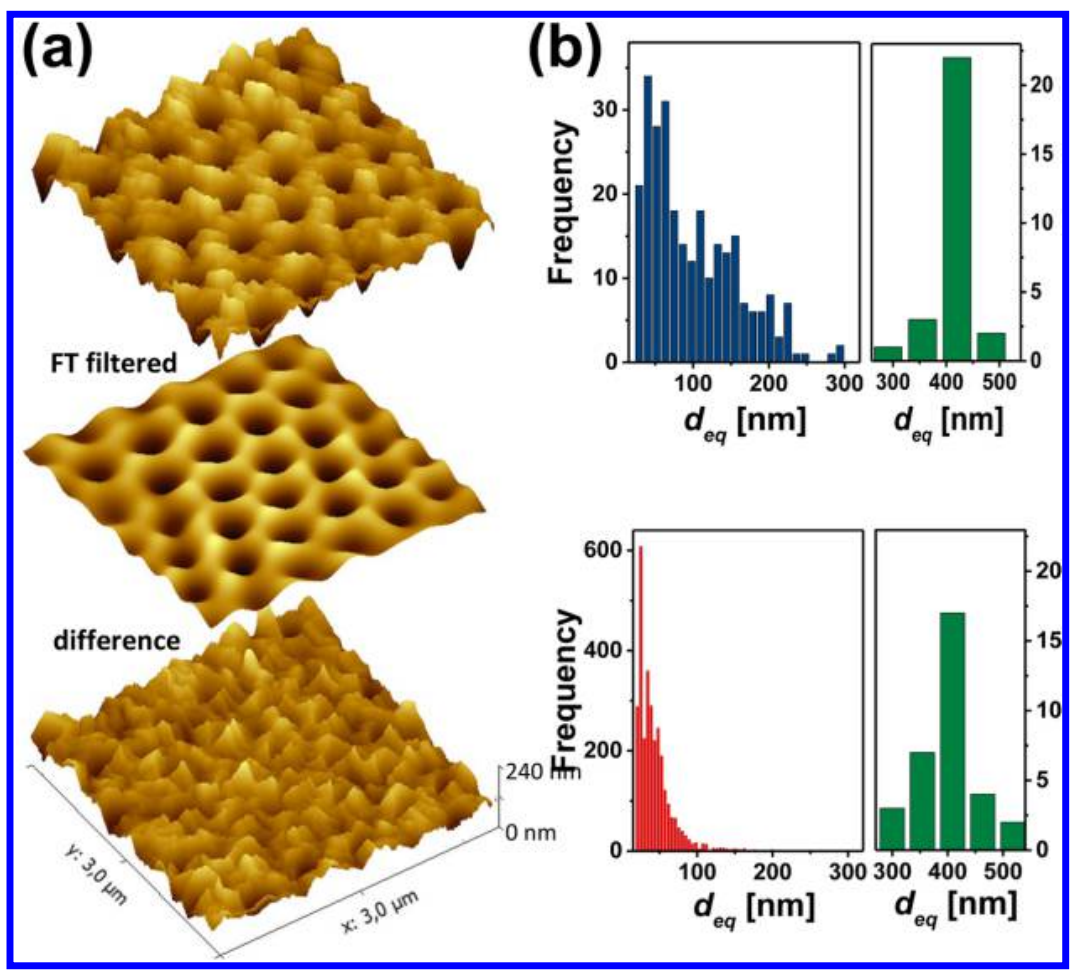

Figure 8. (a) STM image of a roughened $600 \mathrm{~nm} \mathrm{SSV}$ array, $t=0.3$ and $R=3$ (top), filtered to discriminate the small scale roughness (bottom) from the periodic cavity structure (middle). (b) Histograms derived from the filtered STM images, describing the number of features of different sizes observed in the as-grown (top) and the purposely roughened (bottom) SSV arrays. For the small size features, the width of the columns in the rough sample histogram is half that of the untreated one.

such complex and random morphologies, where tractable electromagnetic models ${ }^{48}$ do not capture the full range of possible near-field effects. As a guideline, we may however use the following general conclusions: (i) self-similarity at the subwavelength scale yields a spectrally broad near-field enhancement, independent of the exact morphology (for a given class of substrate); (ii) small features in the $10-50 \mathrm{~nm}$ range $^{47,49}$ yield highest EM enhancement, saturating to a constant value in the quasi-static regime (size « wavelength); ${ }^{48}$ (iii) nanoscale gaps provide the strongest hot-spots, often dominating the SERS response, yet are intrinsically very variable as distance between surface features is poorly controlled or even characterized.

On the other extreme, it is well established that structures appropriately designed and of dimensions of the light wavelength can be developed to perform as antennas to efficiently capture and concentrate light (or reradiate it), much like their microwave or radiofrequency counterparts. ${ }^{50-52}$

To further understand the origin of the observed SERS enhancement of the studied SSV Au arrays, we have thus performed a finer characterization of the surface morphology using high-resolution SEM and STM and image processing techniques. The description of the characteristic dimensions observed in the studied substrates can be more precisely quantified by image processing, as presented in Figure 8 for a $600 \mathrm{~nm}$ SSV array of height $t=0.3$. STM images were processed with Gwyddion software ${ }^{53}$ in order to assess grain distribution in SSV cavities. First, 2D FFT filtering was performed and the hexagonal SSV lattice was subtracted from the original image (see Figure $8 \mathrm{a}$ ). The difference image was masked with a threshold tool and histograms of equivalent diameter $\left(d_{\mathrm{eq}}\right)$ for the grains were obtained (Figure $8 \mathrm{~b}$ ). The same filters were used for both as-grown and roughened SSV arrays. The filtered images clearly confirm our earlier observation, namely, the existence of two qualitatively different length scales. This can be quantified in the histograms shown in Figure $8 \mathrm{~b}$, counting the number of features of different sizes observed in as-grown (top) and purposely roughened (bottom) SSV arrays. Narrow and clear peaks in the histograms appear for structures of equivalent diameter $d_{\mathrm{eq}} \sim 400-450 \mathrm{~nm}$, corresponding to the size of the voids for $t=0.3$ in the ordered cavity template. More significant is the broad distribution of nanostructures of smaller size revealed in the as-grown cavities, peaked around $d_{\mathrm{eq}} \sim 50 \pm 25 \mathrm{~nm}$ and extending up to $\sim 200$ $\mathrm{nm}$, and how this distribution evolves upon electrochemical roughening. The total number of events is a factor 10 larger in the roughened SSV array (corresponding to a relative increase in area $R=3$ ). Moreover, the peak number of events is multiplied by a factor larger than 20 after roughening (accounting for the different binning in the histograms), and the distribution is strongly narrowed and shifted toward smaller sizes centered around $d_{\text {eq }} \sim 20 \pm 20 \mathrm{~nm}$.

We thus conclude that the imperfect or purposely roughened SSV arrays we have described combine two scales that contribute to amplifying the coupling of far-field radiation to the substrate, light-harvesting through wavelength size cavity Mie-like cavity plasmons, and local field enhancement via nanometer-size crevices and protuberances a few tens of nanometers in size. We demonstrated that a residual degree of roughness with consequences in the SERS efficieny is always present in the as-grown structures. The amount and characteristics of this roughness can be strongly augmented by electrochemical roughening, and the mean size of the nanostructures shifted to optimum values for local field enhancement (a trade-off between radiative damping affecting larger sizes and surface scattering affecting the dielectric 
function at the smaller sizes), thus, enhancing the performance of SSV arrays as multiscale antennas.

\section{CONCLUSIONS AND PERSPECTIVES}

We have demonstrated that SSV arrays obtained by electrodeposition present stronger SERS enhancement factors when both nanoscale surface roughness and plasmon modes resulting from the patterned structure are acting in concert. As follows from our study of unpatterned gold, a residual amount of roughness is always present and does not relax, contributing to enhanced Raman signals. These findings are expected to extend to many other SERS substrates, regardless of their fabrication procedure, and should be taken into account when predicting SERS enhancement factors based on simplified, smoother geometries. As we have demonstrated here, this roughness can be augmented and tuned so as to further amplify the SERS efficiency.

This positive interplay may be viewed as the combination of a light-harvesting ability of Mie-type modes, enhancing the interaction between incident light and the gold surface, and the subsequent coupling of these surface-bound plasmon modes with nanoscale protuberances of high curvature that further confine and enhance local electromagnetic fields. While a full model for such a complex system is beyond present day computation capabilities, part of the involved physics can be grasped by a model considering a single void cavity coupled by a $1 \mathrm{~nm}$ gap with a single gold nanoparticle, as described by Huang and co-workers. ${ }^{26}$ As it has been shown from such electromagnetic simulations, all plasmon modes in the cavity are affected by the nanoparticle, but the ones interacting stronger with the nanoparticle are the ones closer to the cavity surface. $^{26}$ In our case, we have not detected new plasmon modes arising from the coupling with nanoscale features (see Figure $6 \mathrm{~b}$ ), presumably because the surface roughness is an integral part of the surface of the cavity, without air gaps. Nevertheless, due to the broad spectral signature of plasmon resonances in SSV arrays, the coupling with nanoscale features of different shapes and sizes, some of them highly delocalized, appears to yield greater enhancements $(\sim 20$-fold $)$ compared to using single uniform nanoparticles $(\sim 10$-fold $){ }^{26}$

We note that this strategy of purposely and weakly roughening far-field light-harvesting antennas to enhance near fields could be potentially applicable to any plasmonic platform. As demonstrated here, a 20 -fold improvement in signal can be achieved with this simple approach, without requiring the more complex combination of different micron and nanosize metallic objects.

Our conclusions are in agreement with, and bring further experimental confirmation to the observations of Hohenau et al. $^{19,20}$ for lithographic substrates, who proposed that a major contribution to the optical near-field and SERS enhancement results from typical edge roughness features when they are localized at regions of large plasmonic mode density.

Our results are also conceptually close to the nanolens concept proposed by Stockman and co-workers, ${ }^{54}$ in the sense that the larger size structures (the SSV cavities in our case) possess an important dipole moment that is efficient to perform as nanoantenna, as an intermediary path to concentrate light in rough structures that further intensify the local field, which by themselves do not have a significant far-field cross section. This is in full agreement with the simulations from Huang et al., ${ }^{26}$ that indicate that more charge accumulates at the junctions in nanoparticle-cavity systems than in nanoparticle-nanoparticle ones. The enhanced charge accumulation is attributed to the large optical cross section of the resonant cavity. A smooth SSV array can indeed perform as a good SERS substrate, and also a nanometer scale nanoparticle can perform as an antenna. The two together, however, performing as a multiscale device work much better in conjunction, each contributing at its optimum regime.

We believe these conclusions define a useful roadmap for the development of optimized plasmonic substrates both for SERS and SPR sensing applications.

\section{METHODS}

Electrochemical Roughening of Unpatterned Gold. Nanostructured Au substrates were prepared from polycrystalline $\mathrm{Au}$ wires (Vega and Camji S.A.I.C, diameter $1 \mathrm{~mm}$ ) following a standard procedure. ${ }^{55}$ Briefly, Au wires were carefully polished with alumina powder $(0.3 \mu \mathrm{m}$, Allied, High Tech Products Inc.), rinsed with Milli-Q water, and dried with nitrogen. A brief annealing process with butane flame was performed in order to systematically reconstruct a smooth surface with an initial area of $\sim 1 \mathrm{~cm}^{2}$. The wire was introduced in a conventional three-electrode glass cell using a saturated calomel electrode (SCE) and high-area platinum foil as reference and counter electrodes, respectively. The $\mathrm{Au}$ wire electrode was then anodized in $0.5 \mathrm{M} \mathrm{H}_{2} \mathrm{SO}_{4}$ solution for 1-15 $\min$ at $2.40 \mathrm{~V}$ (vs SCE) to form a thick hydrous gold oxide layer. By tuning the waiting times at this potential, the final surface roughness of the wire may be controlled. Immediately afterward, the hydrous oxide was electroreduced to metallic $\mathrm{Au}$ by applying a potential sweep from 2.40 to $-0.60 \mathrm{~V}$ at $0.025 \mathrm{~V} /$ s. The Au wire was maintained at the final potential for $5 \mathrm{~min}$ to ensure complete electroreduction of the thick oxide layer. The relative increase in surface area produced after the electrochemical treatment (anodization/reduction cycle) was estimated via the voltammetric roughness factor $R=Q_{f} / Q_{j}$, where $Q_{i}$ and $Q_{f}$ are the charges involved in the $A u$ oxide monolayer electroreduction before and after the anodizing procedure, respectively. The $R$ value was immediately measured in the same electrolyte $\left(0.5 \mathrm{M} \mathrm{H}_{2} \mathrm{SO}_{4}\right)$ used to anodize the $\mathrm{Au}$ electrode by recording a triangular potential sweep from -0.25 to $1.60 \mathrm{~V}$ at a rate $0.1 \mathrm{~V} / \mathrm{s}$. The electroreduction process takes place according to the reaction $\mathrm{AuO}+2 \mathrm{H}^{+}+2 \mathrm{e}^{-} \rightleftharpoons \mathrm{Au}+\mathrm{H}_{2} \mathrm{O}$.

The real surface area $A_{\mathrm{f}}$ of the high area electrode can be obtained by taking into account that for polycrystalline $\mathrm{Au}$ a charge density of $0.42 \mathrm{mC} \cdot \mathrm{cm}^{-2}$ is expected from the reaction, resulting in $A_{\mathrm{f}}=Q_{\mathrm{f}} / 0.42 \mathrm{mC} \cdot \mathrm{cm}^{-2}$, which we link to the relative increase $R=Q_{\mathrm{f}} / Q_{\mathrm{i}}=A_{\mathrm{f}} / A_{\mathrm{i}}$. In particular, we show in Figure 2a the typical voltammograms for $\mathrm{Au}$ substrates in $0.5 \mathrm{M} \mathrm{H}_{2} \mathrm{SO}_{4}$ electrolyte solution before and after the roughening process; in this case, an increase in surface area by a factor of $R=2.5$ is observed.

After the estimation of $A_{\mathrm{f}}$, the electrodes were either (a) removed from the acid solution, rinsed with water, and immersed in a $1 \mathrm{mM}$ MBA (4-mercaptobenzoic acid) ethanolic solution (frozen surfaces) or (b) left exposed in $0.5 \mathrm{M} \mathrm{H}_{2} \mathrm{SO}_{4}$ solution for $60 \mathrm{~min}$, allowing natural relaxation of the surface roughness, and subsequently immersed in the MBA solution (relaxed surfaces). MBA is a widely used nonresonant Raman probe that forms well ordered self-assembled monolayers on $\mathrm{Au}$ with reproducible coverage. Additionally, and as already reported, ${ }^{30}$ thiolate monolayers stabilize the nanostructured $\mathrm{Au}$ by reducing the surface mobility, thus, limiting surface-area decay. 
Fabrication and Roughening of SSV Arrays. To establish evidence for the role of surface roughness in the SERS performance of SSV substrates, we fabricated a number of nominally identical samples made with ordered spheres 600 $\mathrm{nm}$ in diameter, and a stepwise variation of truncation height allowing the tracking and identification of specific plasmon modes. ${ }^{13} \mathrm{SSV}$ arrays were produced according to the procedure detailed in refs 6 and 7. Briefly, polystyrene spheres dispersed in a $1 \%$ water solution (Duke Scientific) are introduced between a glass substrate coated with a $100 \mathrm{~nm}$ gold film (Platypus) and a clean glass slide, with a gap of about $300 \mu \mathrm{m}$. The gold film is previously immersed in a $1 \mathrm{mM}$ cysteamine ethanolic solution overnight to improve the adsorption of polystyrene spheres. During the drying process in an incubation chamber, a sweeping meniscus forms along the substrate, pulling the spheres toward the substrate and into a close-packed hexagonal monolayer. This self-assembly of the template is followed by electrodeposition of Au from a gold salt solution (TG-25 RTU, Technic Inc.), with a deposition rate of $2.5 \mathrm{mC} / \mathrm{min}$. The sample is mounted on a micrometer stage, allowing progressive withdrawing from the solution during the deposition by steps of $500 \mu \mathrm{m}$, yielding discrete levels of truncation parameters for a given sample. ${ }^{56}$ The polystyrene spheres are eventually removed via sonication in a sequence of solvents (dimethylformamide, acetone, ethanol, and water) for 2 min per step.

Annealing of SSV Arrays. By heating the sample at $500{ }^{\circ} \mathrm{C}$ for $90 \mathrm{~min}$, we obtained a reduction of nanoscale surface roughness, while preserving the global structure of the SSV arrays (see Figure 7a). SSV arrays were fabricated as previously described, and cyclic voltammetry in $0.5 \mathrm{M} \mathrm{H}_{2} \mathrm{SO}_{4}$ solution was used to check the decrease in exposed area. The Raman probe 4-mercaptopyridine (4MPy) was deposited onto the samples following the same procedure as MBA described above in order to have a complete and homogeneous monolayer. ${ }^{57}$ SERS measurements were performed on fresh and annealed samples. $4 \mathrm{MPy}$ was electrodesorbed from the substrate before the annealing process and self-assembled again after the annealing. Thermal annealing was performed in a homemade conventional oven, with heating rate set to $10{ }^{\circ} \mathrm{C} \mathrm{min}^{-1}$. After reaching the desired temperature $\left(500{ }^{\circ} \mathrm{C}\right)$, we checked that temperature fluctuations were no greater than $10^{\circ} \mathrm{C}$. We noticed that higher annealing temperatures destroyed the SSV arrays as melted zones could be clearly observed in many parts of the sample.

Substrate Characterization. Reflectivity measurements were used to identify the plasmon modes of the SSV arrays, using a fully automated Wollam WVASE32 variable angle spectroscopic ellipsometer with focusing probes, presenting a $100 \mu \mathrm{m}$ circular spot on the sample with a numerical aperture of $\sim 0.02$. Spectroscopic reflectivity was performed with an angle of incidence of $25^{\circ}$, with a spectral resolution of $5 \mathrm{~nm}$.

The SEM images were recorded with a FEI field-emission gun, Nova NANO-SEM 230, operating at $10 \mathrm{kV}$ and with a tilt angle of $45^{\circ}$. AFM images of the surface were taken with an AFM Veeco Dimension 3100, with MESP tip.

Scanning Tunneling Microscopy (STM) was performed on the samples before and after the annealing process with a Nanoscope IIIA microscope from Digital Instruments (Santa Barbara, CA), in air, in the constant-current mode, and using commercial Pt-Ir tips. Typical tunneling current, bias voltages, and scan rates were $500 \mathrm{pA}, 1 \mathrm{~V}$, and $1-2 \mathrm{~Hz}$, respectively.

SERS measurements were performed using a triple-stage Raman spectrometer (Horiba Jobin-Yvon T64000) operating in subtractive mode, and equipped with a liquid nitrogen-cooled charge-coupled device (CCD). The excitation was done at a fixed wavelength of $647 \mathrm{~nm}$ and $\sim 20 \mathrm{~mW}$ power of an $\mathrm{Ar} / \mathrm{Kr}$ laser focused onto a $\sim 50 \mu \mathrm{m}$ spot. The position on the sample was manually controlled, and the Raman signals were collected in a backscattering configuration with a collection lens of focal length $10 \mathrm{~cm}$. The entrance slit of the spectrometer was kept at $200 \mu \mathrm{m}$, as the Raman peaks for the metal-adsorbed molecule are relatively broad and do not require high spectral resolution. Typical acquisition times were $120 \mathrm{~s}$ for the as-grown SSV substrates and $30 \mathrm{~s}$ for roughened cavities, presenting enhanced signals.

\section{AUTHOR INFORMATION}

\section{Corresponding Author}

*Phone: +54 (294) 4445100. Fax: +54 (294) 4445299. E-mail: afains@cab.cnea.gov.ar.

\section{Present Addresses}

$\S_{\text {The Blackett Laboratory, Department of Physics, Imperial }}$ College London, London SW7 2AZ, United Kingdom (E.C.). "The MacDiarmid Institute for Advanced Materials and Nanotechnology, School of Chemical and Physical Sciences, Victoria University of Wellington, P.O. Box 600, Wellington 6140, New Zealand (B.A.).

\section{Notes}

The authors declare no competing financial interest.

\section{ACKNOWLEDGMENTS}

The authors thank ANPCyT for support through Grants PICT2010 2554, PICT-2012 1808, and PICT-2012 1661. M.E.V. is a member of the research career of CICPBA. A.F. is also a member of CONICET.

\section{REFERENCES}

(1) Maier, S. A. Plasmonics, Fundamentals and Applications; Springer: Berlin, 2007.

(2) Atwater, H. A. The promise of plasmonics. Sci. Am. 2007, 296, $56-63$.

(3) Le Ru, E. C.; Etchegoin, P. G. Principles of Surface Enhanced Raman Spectroscopy and Related Plasmonic Effects; Elsevier: Amsterdam, 2009.

(4) Le Ru, E. C.; Blackie, E.; Meyer, M.; Etchegoin, P. G. SERS enhancement factors: a comprehensive study. J. Phys. Chem. C 2007, 111, 13794-13803.

(5) Meyer, S. A.; Auguié, B.; Le Ru, E. C.; Etchegoin, P. G. Combined SPR and SERS Microscopy in the Kretschmann Configuration. I. Phvs. Chem. A 2012, 116, 1000-1007.

(6) Bartlett, P. N.; Birkin, P. R.; Ghanem, M. A. Electrochemical deposition of macroporous platinum, palladium and cobalt films using polystyrene latex sphere templates. Chem. Commun. 2000, 1671-1672.

(7) Bartlett, P. N.; Baumberg, J. J.; Coyle, S.; Abdelsalam, M. E. Optical properties of nanostructured metal films. Faraday Discuss. 2004, 125, 117.

(8) Kelf, T. A.; Sugawara, Y.; Baumberg, J. J.; Abdelsalam, M.; Bartlett, P. N. Plasmonic band gaps and trapped plasmons on nanostructured metal surfaces. Phys. Rev. Lett. 2005, 95, 116802.

(9) Cintra, S.; Abdelsalam, M. E.; Bartlett, P. N.; Baumberg, J. J.; Kelf, T. A.; Sugawara, Y.; Russell, A. E. Sculpted substrates for SERS. Faradav Discuss. 2006, 132, 191.

(10) Perney, N.; Baumberg, J. J.; Zoorob, M. E.; Charlton, M. D.; Mahnkopf, S.; Netti, C. M. Tuning localized plasmons in nanostructured substrates for surface-enhanced Raman scattering. Opt. Express 2006, 14, 847-857.

(11) Perney, N.; García de Abajo, F.; Baumberg, J.; Tang, A.; Netti, M.; Charlton, M.; Zoorob, M. Tuning localized plasmon cavities for 
optimized surface-enhanced Raman scattering Phys. Rev. B: Condens. Matter Mater. Phys.. 200776.10.1103/PhysRevB.76.035426

(12) Ding, T.; Sigle, D. O.; Herrmann, L. O.; Wolverson, D.; Baumberg, J. J. Nanoimprint Lithography of Al Nanovoids for DeepUV SERS. ACS Appl. Mater. Interfaces 2014, 6, 17358-17363.

(13) Kelf, T.; Sugawara, Y.; Cole, R.; Baumberg, J.; Abdelsalam, M.; Cintra, S.; Mahajan, S.; Russell, A.; Bartlett, P. Localized and delocalized plasmons in metallic nanovoids Phys. Rev. B: Condens. Matter Mater. Phys.. 200674.10.1103/PhysRevB.74.245415

(14) Cole, R. M.; Baumberg, J. J.; Garcia de Abajo, F. J.; Mahajan, S.; Abdelsalam, M.; Bartlett, P. N. Understanding Plasmons in Nanoscale Voids. Nano Lett. 2007, 7, 2094-2100.

(15) Lacharmoise, P.; Tognalli, N.; Goñi, A.; Alonso, M.; Fainstein, A.; Cole, R.; Baumberg, J.; Garcia de Abajo, J.; Bartlett, P. Imaging optical near fields at metallic nanoscale voids Phys. Rev. B: Condens. Matter Mater. Phys.. 200878.10.1103/PhysRevB.78.125410

(16) Mahajan, S.; Cole, R. M.; Soares, B. F.; Pelfrey, S. H.; Russell, A. E.; Baumberg, J. J.; Bartlett, P. N. Relating SERS Intensity to Specific Plasmon Modes on Sphere Segment Void Surfaces. J. Phys. Chem. C 2009, 113, 9284-9289.

(17) Schmidt, M.; Tognalli, N. G.; Otte, M. A.; Alonso, M. I.; Sepúlveda, B.; Fainstein, A.; Goñi, A. R. Spatial Distribution of Optical Near-Fields in Plasmonic Gold Sphere Segment Voids. Plasmonics 2013, 8, 921-930.

(18) Tognalli, N. G.; Fainstein, A.; Calvo, E. J.; Abdelsalam, M.; Bartlett, P. N. Incident Wavelength Resolved Resonant SERS on Au Sphere Segment Void (SSV) Arrays. J. Phys. Chem. C 2012, 116, 3414-3420.

(19) Sow, I.; Grand, J.; Lévi, G.; Aubard, J.; Félidj, N.; Tinguely, J.-C.; Hohenau, A.; Krenn, J. R. Revisiting Surface-Enhanced Raman Scattering on Realistic Lithographic Gold Nanostripes. J. Phys. Chem. C 2013, 117, 25650-25658.

(20) Trügler, A.; Tinguely, J.-C.; Jakopic, G.; Hohenester, U.; Krenn, J. R.; Hohenau, A. Near-field and SERS enhancement from rough plasmonic nanoparticles Phys. Rev. B: Condens. Matter Mater. Phys. 201489.10.1103/PhysRevB.89.165409

(21) Kern, A. M.; Martin, O. J. F. Excitation and Reemission of Molecules near Realistic Plasmonic Nanostructures. Nano Lett. 2011, 11, 482-487.

(22) Kern, A. M.; Meixner, A. J.; Martin, O. J. F. MoleculeDependent Plasmonic Enhancement of Fluorescence and Raman Scattering near Realistic Nanostructures. ACS Nano 2012, 6, 98289836.

(23) Leordean, C.; Potara, M.; Boca-Farcau, S.; Vulpoi, A.; Astilean, S.; Farcau, C. Multiscale electromagnetic SERS enhancement on selfassembled micropatterned gold nanoparticle films. J. Raman Spectrosc. 2014, 45, 627-635.

(24) Scodeller, P.; Flexer, V.; Szamocki, R.; Calvo, E. J.; Tognalli, N.; Troiani, H.; Fainstein, A. Wired-Enzyme Core-Shell Au Nanoparticle Biosensor. I. Am. Chem. Soc. 2008, 130, 12690-12697.

(25) Tognalli, N. G.; Scodeller, P.; Flexer, V.; Szamocki, R.; Ricci, A.; Tagliazucchi, M.; Calvo, E. J.; Fainstein, A. Redox molecule based SERS sensors. Phys. Chem. Chem. Phys. 2009, 11, 7412-7423.

(26) Huang, F. M.; Wilding, D.; Speed, J. D.; Russell, A. E.; Bartlett, P. N.; Baumberg, J. J. Dressing Plasmons in Particle-in-Cavity Architectures. Nano Lett. 2011, 11, 1221-1226.

(27) Salvarezza, R. C.; Arvia, A. J. In Modern Aspects of Electrochemistry; Conway, B. E., Bockris, J. O., White, R. E., Eds.; Springer: New York, 1996; Vol. 28, pp 289-373.

(28) Vela, M. E.; Zerbino, J. O.; Arvia, A. J. Ellipsometric study of hydrous gold oxide layers and gold surfaces resulting from their electroreduction. Thin Solid Films 1993, 233, 82-85.

(29) Macchi, C.; Somoza, A.; Mariazzi, S.; Brusa, R. S.; Vericat, C.; Vela, M. E.; Salvarezza, R. C. Characterization of high area nanostructured gold surfaces by slow positron depth profiling. Phys. Status Solidi C 2009, 6, 2585-2588.

(30) Vericat, C.; Benitez, G. A.; Vela, M. E.; Salvarezza, R. C.; Tognalli, N. G.; Fainstein, A. Restricted Surface Mobility of Thiolate-
Covered Metal Surfaces: A Simple Strategy to Produce High-Area Functionalized Surfaces. Langmuir 2007, 23, 1152-1159.

(31) Michota, A.; Bukowska, J. Surface-enhanced Raman scattering (SERS) of 4- mercaptobenzoic acid on silver and gold substrates. J. Raman Spectrosc. 2003, 34, 21-25.

(32) Karim, S.; Toimil-Molares, M. E.; Balogh, A. G.; Ensinger, W.; Cornelius, T. W.; Khan, E. U.; Neumann, R. Morphological evolution of $\mathrm{Au}$ nanowires controlled by Rayleigh instability. Nanotechnology 2006, 17, 5954-5959.

(33) Sun, Y.; Mayers, B.; Xia, Y. Transformation of silver nanospheres into nanobelts and triangular nanoplates through a thermal process. Nano Lett. 2003, 3, 675-679.

(34) Mullins, W. W. Theory of thermal grooving. J. Appl. Phys. 1957, $28,333-339$.

(35) Mullins, W. W. Flattening of a nearly plane solid surface due to capillarity. I. Appl. Phvs. 1959, 30, 77-83.

(36) Andreasen, G.; Schilardi, P. L.; Azzaroni, O.; Salvarezza, R. C. Thermal Annealing of Patterned Metal Surfaces. Langmuir 2002, 18, 10430-10434.

(37) Schwoebel, R. L.; Shipsey, E. J. Step Motion on Crystal Surfaces. I. Appl. Phys. 1966, 37, 3682-3686.

(38) Ehrlich, G.; Hudda, F. G. Atomic View of Surface Self Diffusion: Tungsten on Tungsten. J. Chem. Phys. 1966, 44, 1039-1049.

(39) Rost, M. J. In Situ Real-Time Observation of Thin Film Deposition: Roughening, Zeno Effect, Grain Boundary Crossing Barrier, and Steering. Phvs. Rev. Lett. 2007, 99, 266101.

(40) Haynes, C. L.; Yonzon, C. R.; Zhang, X.; Van Duyne, R. P. Surface-enhanced Raman sensors: early history and the development of sensors for quantitative biowarfare agent and glucose detection. J. Raman Spectrosc. 2005, 36, 471-484.

(41) McQuillan, A. J. The discovery of surface-enhanced Raman scattering. Notes Rec. R. Soc. 2009, 63, 105-109.

(42) Moskovits, M. Surface-enhanced spectroscopy. Rev. Mod. Phvs. 1985, 57, 783.

(43) Moskovits, M. How the localized surface plasmon became linked with surface-enhanced Raman spectroscopy. Notes Rec. R. Soc. 2012, 66, 195-203.

(44) Brown, R. J. C.; Wang, J.; Milton, M. J. T. Electromagnetic Modelling of Raman Enhancement from Nanoscale Structures as a Means to Predict the Effcacy of SERS Substrates. J. Nanomater 2007, 2007, 1-10.

(45) Wang, H.; Goodrich, G. P.; Tam, F.; Oubre, C.; Nordlander, P.; Halas, N. J. Controlled Texturing Modifies the Surface Topography and Plasmonic Properties of Au Nanoshells. J. Phys. Chem. B 2005, 109, 11083-11087.

(46) Rodríguez-Fernández, J.; Funston, A. M.; Pérez-Juste, J.; Álvarez-Puebla, R. A.; Liz-Marzán, L. M.; Mulvaney, P. The effect of surface roughness on the plasmonic response of individual sub-micron gold spheres. Phvs. Chem. Chem. Phvs. 2009, 11, 5909.

(47) Brolo, A. G.; Irish, D. E.; Szymanski, G.; Lipkowski, J. Relationship between SERS intensity and both surface coverage and morphology for pyrazine adsorbed on a polycrystalline gold electrode. Langmuir 1998, 14, 517-527.

(48) García-Vidal, F. J.; Pendry, J. B. Collective theory for surface enhanced Raman scattering. Phvs. Rev. Lett. 1996, 77, 1163.

(49) Douketis, C.; Haslett, T. L.; Wang, Z.; Moskovits, M.; Iannotta, S. Self-affne silver films and surface-enhanced Raman scattering: Linking spectroscopy to morphology. I. Chem. Phys. 2000, 113, 11315.

(50) Kosako, T.; Kadoya, Y.; Hofmann, H. F. Directional control of light by a nano-optical Yagi-Uda antenna. Nat. Photonics 2010, 4, 312315 .

(51) Dregely, D.; Taubert, R.; Dorfmüller, J.; Vogelgesang, R.; Kern, K.; Giessen, H. 3D optical Yagi-Uda nanoantenna array. Nat. Commun. 2011, 2, 267.

(52) Novotny, L.; Van Hulst, N. Antennas for light. Nat. Photonics 2011, 5, 83-90.

(53) Nečas, D.; Klapetek, P. Gwyddion: an open-source software for SPM data analysis. Cent. Eur. I. Phvs. 2012, 10, 181-188. 
(54) Li, K.; Stockman, M. I.; Bergman, D. J. Self-Similar Chain of Metal Nanospheres as an Efficient Nanolens. Phys. Rev. Lett. 2003, 91, 227402.

(55) Cortés, E.; Rubert, A. A.; Benitez, G.; Carro, P.; Vela, M. E.; Salvarezza, R. C. Enhanced Stability of Thiolate Self-Assembled Monolayers (SAMs) on Nanostructured Gold Substrates. Langmuir 2009, 25, 5661-5666.

(56) Tognalli, N. G.; Cortés, E.; Hernández-Nieves, A. D.; Carro, P.; Usaj, G.; Balseiro, C. A.; Vela, M. E.; Salvarezza, R. C.; Fainstein, A. From Single to Multiple Ag-Layer Modification of $\mathrm{Au}$ Nanocavity Substrates: A Tunable Probe of the Chemical Surface-Enhanced Raman Scattering Mechanism. ACS Nano 2011, 5, 5433-5443.

(57) Ramírez, E. A.; Cortés, E.; Rubert, A. A.; Carro, P.; Benítez, G.; Vela, M. E.; Salvarezza, R. C. Complex Surface Chemistry of 4Mercaptopyridine Self-Assembled Monolayers on $\mathrm{Au}(111)$. Langmuir 2012, 28, 6839-6847. 